\title{
Mining Small Routine Clinical Data: A Population Pharmacokinetic Model and Optimal Sampling Times of Capecitabine and its Metabolites.
}

\author{
Esther Oyaga-Iriarte ${ }^{1}$, Asier Insausti ${ }^{1}$, Lorea Bueno $^{1}$, Onintza Sayar ${ }^{1}$, Azucena Aldaz $^{2}$ \\ 1. Pharmamodelling S.L., Pamplona, Spain. ${ }^{2}$ Service of Hospital Pharmacy, Clínica Universidad de Navarra, Pío XII 36 , \\ Pamplona, Spain.
}

Received, January 31, 2019; Revised, February 27, 2019; Accepted, April 1, 2019; Published, April 3, 2019.

\begin{abstract}
Purpose: The present study was performed to demonstrate that small amounts of routine clinical data allow to generate valuable knowledge. Concretely, the aims of this research were to build a joint population pharmacokinetic model for capecitabine and three of its metabolites (5-DFUR, 5-FU and 5-FUH2) and to determine optimal sampling times for therapeutic drug monitoring. Methods: We used data of 7 treatment cycles of capecitabine in patients with metastatic colorectal cancer. The population pharmacokinetic model was built as a multicompartmental model using NONMEM and was internally validated by visual predictive check. Optimal sampling times were estimated using PFIM 4.0 following D-optimality criterion. Results: The final model was a multicompartmental model which represented the sequential transformations from capecitabine to its metabolites 5-DFUR, 5-FU and 5-FUH2 and was correctly validated. The optimal sampling times were $0.546,0.892,1.562,4.736$ and 8 hours after the administration of the drug. For its correct implementation in clinical practice, the values were rounded to $0.5,1,1.5,5$ and 8 hours after the administration of the drug. Conclusions: Capecitabine, 5-DFUR, 5-FU and 5-FUH2 can be correctly described by the joint multicompartmental model presented in this work. The aforementioned times are optimal to maximize the information of samples. Useful knowledge can be obtained for clinical practice from small databases.
\end{abstract}

\section{INTRODUCTION}

Capecitabine is an oral prodrug of 5-fluorouracil (5-FU) used against numerous tumors such as colorectal cancer, among others (1-3). The active metabolite 5-FU, through its own metabolites, acts on DNA synthesis and inhibits the formation of RNA, producing a cellular imbalance that results in cell death (4).

Capecitabine is converted principally in the liver to 5'-deoxy-5-fluorocytidine (5-DFCR), which is converted to 5'-deoxy-5-fluorouridine (5DFUR) in the liver and tumor tissues. Then, 5DFUR is metabolized to 5-FU by thymidine phosphorylase, an enzyme that has higher concentrations in tumor tissues (5). In a second stage, 5-FU is catalyzed to dihydro-5-fluorouracil (5-FUH2), $\alpha$-fluoro-ureidopropionic acid (FUPA) and, finally, $\alpha$-fluoro- $\beta$-alanine (FBAL) (6).

The therapeutic drug monitoring (TDM) of capecitabine is recommended specially for geriatric patients and those with hepatic impairment $(4,7,8)$. Furthermore, the individualization of the treatment is fundamental to achieve an optimal pharmacotherapy, maximizing its efficacy and reducing adverse effects (9). Hence, the joint pharmacokinetic study of this prodrug and its metabolites is necessary. The pharmacokinetics (PK) of this drug have been characterized by other authors using different compartmental models (10-12). The construction of this population PK model, followed by the application of Bayesian methods (13) and simulation techniques (14) permit the individualization of the dosing of capecitabine and the optimization of the treatment, as described in other works $(15,16)$.

The accuracy of PK models is closely related to the quality of the information retrieved from blood samples. However, to our knowledge, optimal sampling times for this drug have not been estimated in the literature. The correct collection of blood samples allows to maximize subsequent pharmacokinetic analyses $(17,18)$ and to minimize the number of extractions.

This work aimed at building a joint population pharmacokinetic model for capecitabine, 5-DFUR, 5-FU and 5-FUH2 and at determining optimal sampling times for TDM. The fulfillment of these objectives demonstrates that even small amounts of routine clinical data generate valuable knowledge for clinical practice.

Corresponding Author: Esther Oyaga-Iriarte, Pharmamodelling S.L., Pamplona, Spain; E-mail address: eoyaga@pharmamodelling.com 


\section{MATERIALS AND METHODS}

\section{Study population.}

The data, which came from routine clinical practice, consisted of plasma concentrations of capecitabine, 5-DFUR, 5-FU and 5-FUH2 from seven treatment cycles of seven patients with metastatic colorectal cancer. They received a dose of $825 \mathrm{mg} / \mathrm{m}^{2}$ of capecitabine every 12 hours and $60 \mathrm{mg} / \mathrm{m}^{2}$ of oxaliplatin in 1 hour during 14 days, followed by a rest of 7 days. During the 12 hours after capecitabine administration, between 7-10 blood extractions were collected from each patient and they were frozen at $-70^{\circ} \mathrm{C}$ until further analysis. The quantification of the samples followed high-performance liquid chromatography (HPLC), described by Zufia et al. (19).

\section{Population pharmacokinetic modelling.} Capecitabine, 5-DFUR, 5-FU and 5-FUH2 plasma concentrations were modeled by multicompartment models using non-linear mixed effects modelling (NONMEM) (20).

Every compartment was parameterized in terms of volume of distribution and clearance. The model was built stepwise. First, capecitabine was characterized testing with mono, bi and tricomparmental models. Then, 5-DFUR was included and tested following the same criteria. Lastly, we proceeded analogously with 5-FU and 5-FUH2. Intersubject variabilities (ISV) were tested for all PK parameters using an exponential error model and intrasubject variabilities (EPS) were tested using the combined residual error. The inclusion of covariates was not recommended for such small samples, as they are prone to selectionbias, causing overlearning of the model, and may falsely appear to be clinically relevant (21-23).

The evaluation of our model was made in accordance to the values given by the objective function (OF=-2log_likelihood), the Akaike information criterion (AIC), Bayesian information criterion (BIC) and the coefficient of determination of linear regression of the observed versus predicted values. Additionally, different diagnostic plots were used to visually evaluate the model: observed versus population and versus individual predicted values and weighted residuals versus time, versus population and versus individual predicted concentrations.

Visual predictive check (VPC) with 1000 Monte Carlo simulations was carried out to validate the final model (24).

Table 1. Demographic, anthropometric and basal analytical data.

\begin{tabular}{lcc}
\hline \multicolumn{1}{c}{ Patient characteristics } & Median value & Min-Max \\
\hline Age (years) & 47.00 & $32.00-77.00$ \\
Body surface $\left(\mathrm{m}^{2}\right)$ & 1.95 & $1.71-2.14$ \\
CEA (ng/mL) & 9.10 & $2.10-2598.2$ \\
AST (UI/L) & 17.00 & $6.00-175.00$ \\
ALT (UI/L) & 14.00 & $4.00-100.00$ \\
ALP (UI/L) & 133.00 & $94.00-1785.00$ \\
GGTP (UI/L) & 39.50 & $16.00-850.00$ \\
DBil (mg/dL) & 0.19 & $0.03-5.80$ \\
TBil (mg/dL) & 0.70 & $0.36-8.27$ \\
LDH (UI/L) & 212.00 & $180.00-1299.00$ \\
SCr (mg/dL) & 1.00 & $0.70-4.30$ \\
Hb (g/dL) & 11.20 & $8.30-15.50$ \\
Ht (\%) & 32.25 & $25.00-45.00$ \\
MCV (fL) & 82.60 & $75.70-92.00$ \\
MCH (pg) & 28.20 & $25.20-30.80$ \\
Platelets (/pL) & 214.00 & $196.00-502.00$ \\
Leukocytes $/$ pL) & 7.40 & $5.60-38.50$ \\
Neutrophils (/pL) & 4.83 & $3.30-31.93$ \\
Lymphocytes (/pL) & 1.62 & $1.44-2.52$ \\
Monocytes (/pL) & 0.74 & $0.59-2.89$ \\
Eosinophils (/pL) & 0.19 & $0.08-0.92$ \\
Basophils (/pL) & 0.07 & $0.05-0.35$ \\
CEA: Carcinoembryonic antigen. AST: Aspartate aminotransferase. ALT: Alanine aminotransferase. ALP: Alkaline \\
phosphatase. GGTP: Gamma glutamyltranspeptidase. DBil: Direct bilirubin. TBil: Total bilirubin. LDH: Lactate \\
dehydrogenase. SCr: Serum creatinine. Hb: Hemoglobin. Ht: Hematocrit. MCV: Mean corpuscular volume. MCH: \\
Mean corpuscular hemoglobin. & & \\
\hline
\end{tabular}


Optimal sampling times.

Optimal times were estimated according to Doptimality criterion, using PFIM Interface 4.0 (25). The model employed in this process was designed to prioritize the correct adjustment of the active metabolite, 5-FU, using the same methodology as in the joint population PK model.

Monte Carlo simulations, used to perform VPC, allowed to visually validate the adequacy of the established optimal sampling times.

\section{RESULTS}

A total of 227 samples were analyzed, of which 63 corresponded to capecitabine, 69 to 5-DFUR, 47 to 5-FU and 48 to 5-FUH2. Table 1 summarizes the characteristics of the population of the study.

\section{Pharmacokinetic final model.}

The final population pharmacokinetic model that best described capecitabine and its metabolites is shown in Figure 1. This model converged to a nadir in the OF with a minimum value of -368.683 .

In this multicompartmental model, $m$ denoted the amount of drug in the dosing compartment. Capecitabine was represented by a single compartment $\left(A_{1}\right)$, parameterized by the central volume of distribution $\left(V_{1}\right)$, an absorption rate constant $\left(k_{\mathrm{a}}\right)$, a lag time $\left(t_{\mathrm{lag}}\right)$, a clearance $\left(C l_{0}\right)$ and the clearance of the compartment to the metabolite 5-DFUR ( $\left.\mathrm{Cl}_{\text {cap }}\right)$. 5-DFUR was described by two compartments $\left(A_{2}\right.$ and $\left.A_{3}\right)$, in which the central and peripheral distribution volumes of 5-DFUR $\left(V_{2}, V_{3}\right.$, respectively), the flow between both compartments $\left(\mathrm{Cl}_{23}\right.$ and $\left.\mathrm{Cl}_{32}\right)$ and the clearance of the central compartment to the metabolite 5-FU ( $\left.\mathrm{Cl}_{5 \text {-DFUR }}\right)$ were used. 5-FU was described by a single compartment $\left(A_{4}\right)$, parameterized by the central volume of distribution $\left(V_{4}\right)$ and the clearance of the compartment to the last characterized metabolite, $5-\mathrm{FUH}_{2},\left(\mathrm{Cl}_{5-\mathrm{FU}}\right)$. The metabolite 5-FUH2 was represented by a single compartment $\left(A_{5}\right)$, the parameterization used consisted of the central distribution volume of 5-FUH2 $\left(V_{5}\right)$ and the clearance of 5-FUH $2\left(\mathrm{Cl}_{5}-\mathrm{FUH} 2\right)$. Consequently, the following equations described this multicompartmental model:

$$
\frac{d m}{d t}=-k_{\mathrm{a}} m
$$

$$
\frac{d A_{1}}{d t}=k_{\mathrm{a}} m-\frac{C l_{\text {cap }}}{V_{1}} A_{1}-\frac{C l_{0}}{V_{1}} A_{1},
$$

$$
\begin{gathered}
\frac{d A_{2}}{d t}=\frac{C l_{\text {cap }}}{V_{1}} A_{1}-\frac{C l_{23}}{V_{2}} A_{2}+\frac{C l_{32}}{V_{3}} A_{3}-\frac{C l_{5-\mathrm{DFUR}}}{V_{2}} A_{2} \\
\frac{d A_{3}}{d t}=\frac{C l_{23}}{V_{2}} A_{2}-\frac{C l_{32}}{V_{3}} A_{3} \\
\frac{d A_{4}}{d t}=\frac{C l_{5-\mathrm{DFUR}}}{V_{2}} A_{2}-\frac{C l_{5-\mathrm{FU}}}{V_{4}} A_{4} \\
\frac{d A_{5}}{d t}=\frac{C l_{5-\mathrm{FU}}}{V_{4}} A_{4}-\frac{C l_{5-\mathrm{FUH} 2}}{V_{5}} A_{5} .
\end{gathered}
$$

Population pharmacokinetic parameters of capecitabine and its metabolites can be seen in Table 2.

Individual predictions of capecitabine, 5DFUR, 5-FU and 5-FUH2 are shown in Figure 2. $\mathrm{R}$ squared values of each of the linear regression were $0.873,0.810,0.886$ and 0.571 , respectively. Capecitabine $\mathrm{R}$ squared value was conditioned by a single point (observed value 7.87 and individual predicted value 7.43 ). Omitting this point the $\mathrm{R}$ squared value was 0.374 .

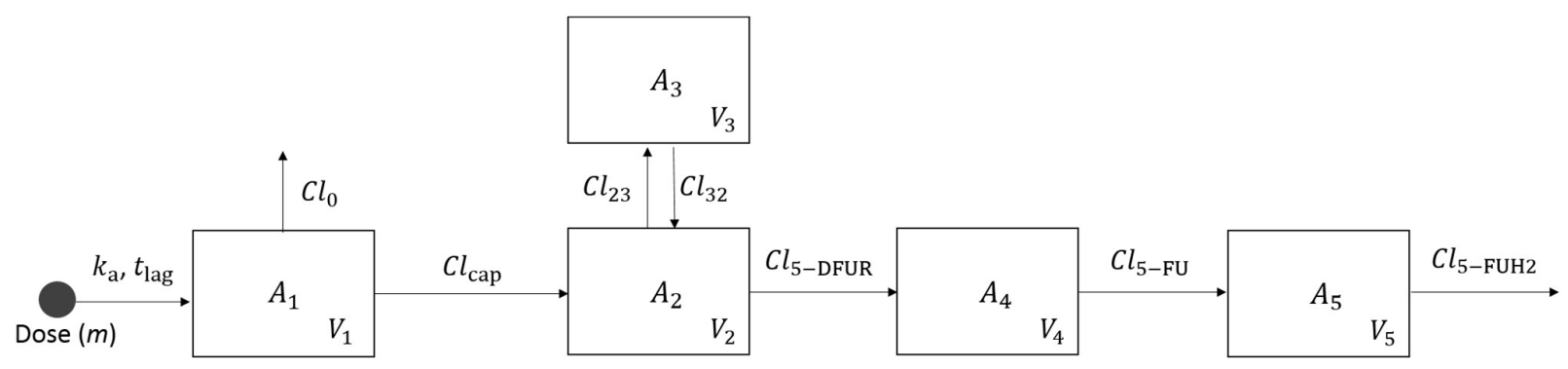

Figure 1. Scheme of the joint pharmacokinetic model for capecitabine, 5-DFUR, 5-FU and 5-FUH2. 
Table 2. Population pharmacokinetic parameters of capecitabine, 5-DFUR, 5-FU and 5-FUH2.

\begin{tabular}{|c|c|c|c|}
\hline Parameter & Estimate & RSE (\%) & CI 95 \\
\hline$k_{\mathrm{a}}\left(\mathrm{h}^{-1}\right)$ & 0.995 & 7.39 & $(0.85-1.14)$ \\
\hline$t_{\text {lag }}(\mathrm{h})$ & 0.0172 & 87.79 & $(0.0001-0.047)$ \\
\hline$C l_{\text {cap }}(\mathrm{L} / \mathrm{h})$ & 4.05 & 20.07 & $2.42-5.68$ \\
\hline$C l_{0}(\mathrm{~L} / \mathrm{h})$ & 396 & 10.02 & $(316.6-475.4)$ \\
\hline$V_{1}(\mathrm{~L})$ & 423 & 12.76 & $(315-531)$ \\
\hline$C l_{5-\mathrm{DFUR}}(\mathrm{L} / \mathrm{h})$ & 2.56 & 21.29 & $(1.47-3.65)$ \\
\hline$V_{2}(\mathrm{~L})$ & 1 FIX & - & - \\
\hline$V_{3}(\mathrm{~L})$ & 1 FIX & - & - \\
\hline$C l_{23}(\mathrm{~L} / \mathrm{h})$ & 3.42 & 73.39 & $(0.0001-8.44)$ \\
\hline$C l_{32}(\mathrm{~L} / \mathrm{h})$ & 0.626 & 53.83 & $(0.0001-1.3)$ \\
\hline$C l_{5-\mathrm{FU}}(\mathrm{L} / \mathrm{h})$ & 10.9 & 31.84 & (3.96-17.84) \\
\hline$V_{4}(\mathrm{~L})$ & 1 FIX & - & - \\
\hline$C l_{5-\mathrm{FUH} 2}(\mathrm{~L} / \mathrm{h})$ & 2.2 & 19.95 & $(1.32-3.08)$ \\
\hline$V_{5}(\mathrm{~L})$ & 1 FIX & - & - \\
\hline ISV $k_{\mathrm{a}}(\%)$ & 101.49 & 85.53 & $(0.0001-2.79)$ \\
\hline ISV $t_{\text {lag }}(\%)$ & 233.67 & 76.92 & $(0.0001-13.86)$ \\
\hline ISV $C l_{\text {cap }}(\%)$ & 39.87 & 58.36 & $(0.0001-0.34)$ \\
\hline $\mathrm{ISVCl}_{0}(\%)$ & - & - & - \\
\hline ISV $V_{1}(\%)$ & 99.89 & 94.69 & $(0.0001-0.34)$ \\
\hline ISV $C l_{5-\text { DFUR }}(\%)$ & - & - & - \\
\hline EPS1 $(\%)$ & 80.56 & 22.34 & $(0.359-0.94)$ \\
\hline EPS2 (\%) & 4.21 & 86.11 & $(0.00001-0.00127)$ \\
\hline EPS3 (\%) & 60.17 & 21.35 & $(0.21-0.52)$ \\
\hline EPS4 (\%) & - & - & - \\
\hline EPS5 (\%) & 41.35 & 47.6 & $(0.0082-0.334)$ \\
\hline EPS6 (\%) & 27.52 & 37.1 & $(0.000436-0.003)$ \\
\hline EPS7 (\%) & 33.62 & 128 & $(0.0001-0.40)$ \\
\hline EPS8 (\%) & 50.96 & 43.42 & $(0.03-0.36)$ \\
\hline
\end{tabular}

RSE: Residual standard error. CI: Confidence interval. ISV: Intersubject variability. EPS1: Proportional error of the combined capecitabine residual error. EPS2: Additive error of the combined capecitabine residual error. EPS3: Proportional error of the combined 5-DFUR residual error. EPS4: Additive error of the combined 5-DFUR residual error. EPS5: Proportional error of the combined 5-FU residual error. EPS6: Additive error of the combined 5-FU residual error. EPS7: Proportional error of the combined 5-FUH2 residual error. EPS8: Additive error of the combined 5-FUH2 residual error.

Using the final model, 1000 Monte Carlo simulations of individual concentration over time profiles for capecitabine, 5-DFUR, 5-FU and 5FUH2 were carried out following VPC scheme (Figure 5). A high percentage of the raw data was included in the percentile 5 and 95 range of the simulations. Specifically, the percentage of points that fell within the simulated range was $95.24 \%$, $98.55 \%, 87.23 \%$ and $93.75 \%$ for capecitabine, 5DFUR, 5-FU and 5-FUH2, respectively.

\section{Optimal sampling times.}

The model of the active metabolite, 5-FU, used for the estimation of optimal sampling times was a monocompartmental model parameterized by the volume of distribution $(V)$, first order constant rate $\left(k_{\mathrm{a}}\right)$ and clearance $(\mathrm{Cl})$. Mean (and interpatient variance) population parameters were: $V=5.97 \times 10^{2}$ (3.31) L, $k_{\mathrm{a}}=2.59 \times 10^{-2} \quad\left(1 \times 10^{-6}\right) \quad \mathrm{h}^{-1}$ and $C l=9.91 \times 10^{1}\left(1 \times 10^{-6}\right) \mathrm{L} / \mathrm{h}$. VPC validation on this model resulted in $91.49 \%$ of the 5 -FU samples within the $90 \%$ confidence interval.

This model was used to calculate optimal sampling times using PFIM. The results obtained for 5-FU were: $0.546,0.892,1.562,4.736$ and 8 hours after capecitabine administration. The value of D-optimality criterion was $5.9940 \times 10^{-3}$.

Moreover, Monte Carlo simulations using the final joint model permitted to visually validate the adequacy of the computed optimal sampling times. Figure 6 shows the population PK profiles of the four molecules and, in vertical lines, the computed optimal sampling times. 

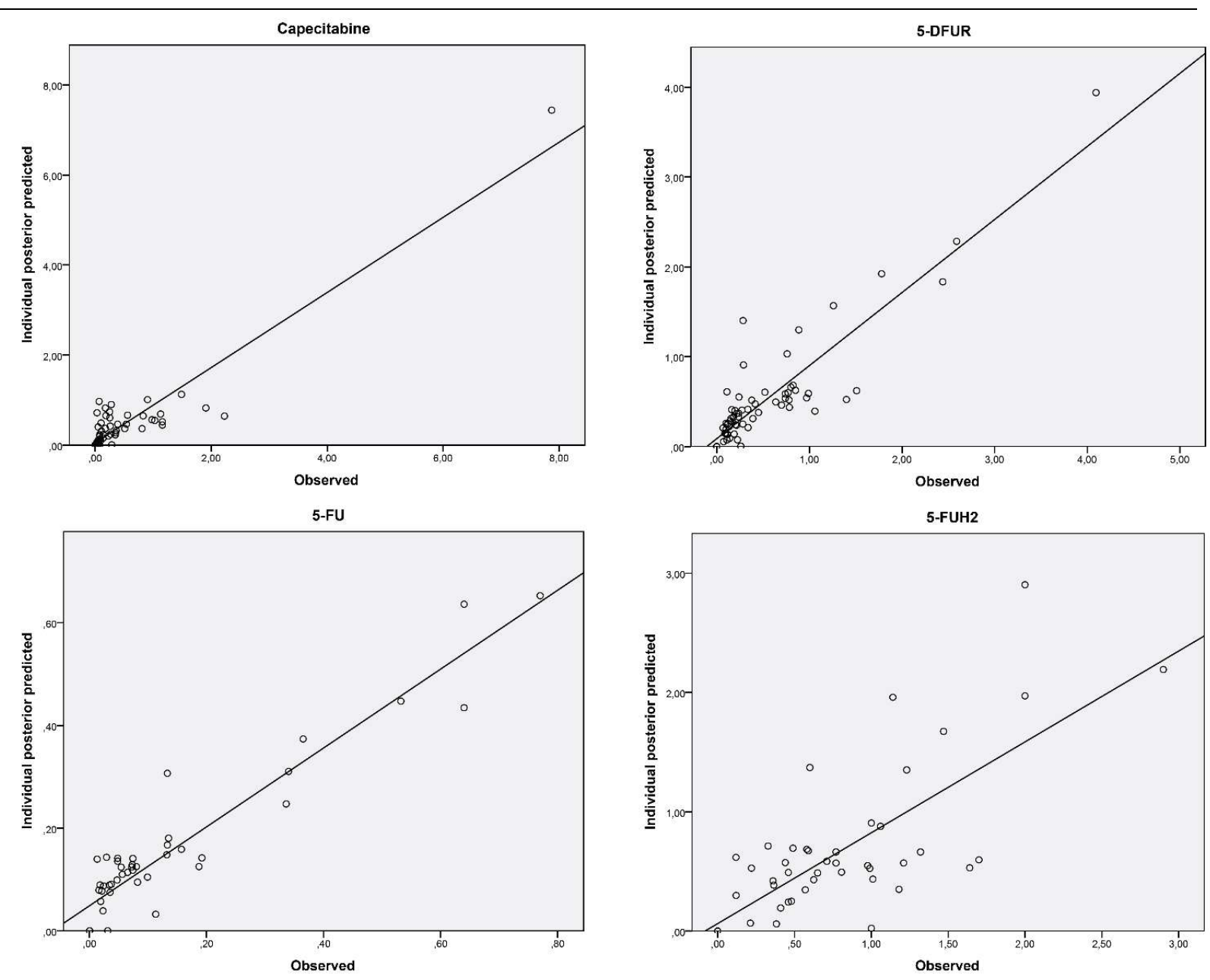

Figure 2. Individual predictions by the joint model for capecitabine, 5-DFUR, 5-FU and 5-FUH2.

Scatter plots of weighted residuals versus time, versus population predicted concentrations and versus individual predicted concentrations are shown in Figure 3.
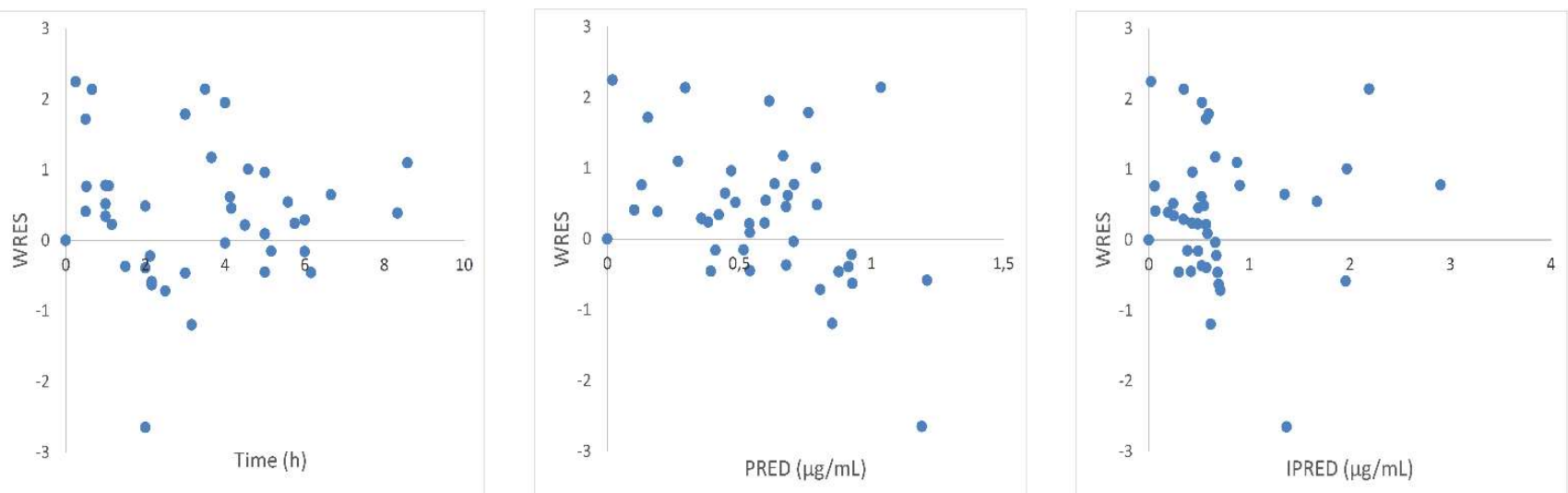

Figure 3. Weighted residuals versus time (left), weighted residuals versus population predicted concentrations (center) and weighted residuals versus individual predicted concentrations (right). WRES: weighted residuals. PRED: population predicted concentrations. IPRED: individual predicted concentrations. 
Concentration-time profiles of the observations and predictions of capecitabine, 5-DFUR, 5-FU and 5-FUH2 are shown in Figure 4 for each of the seven patients.
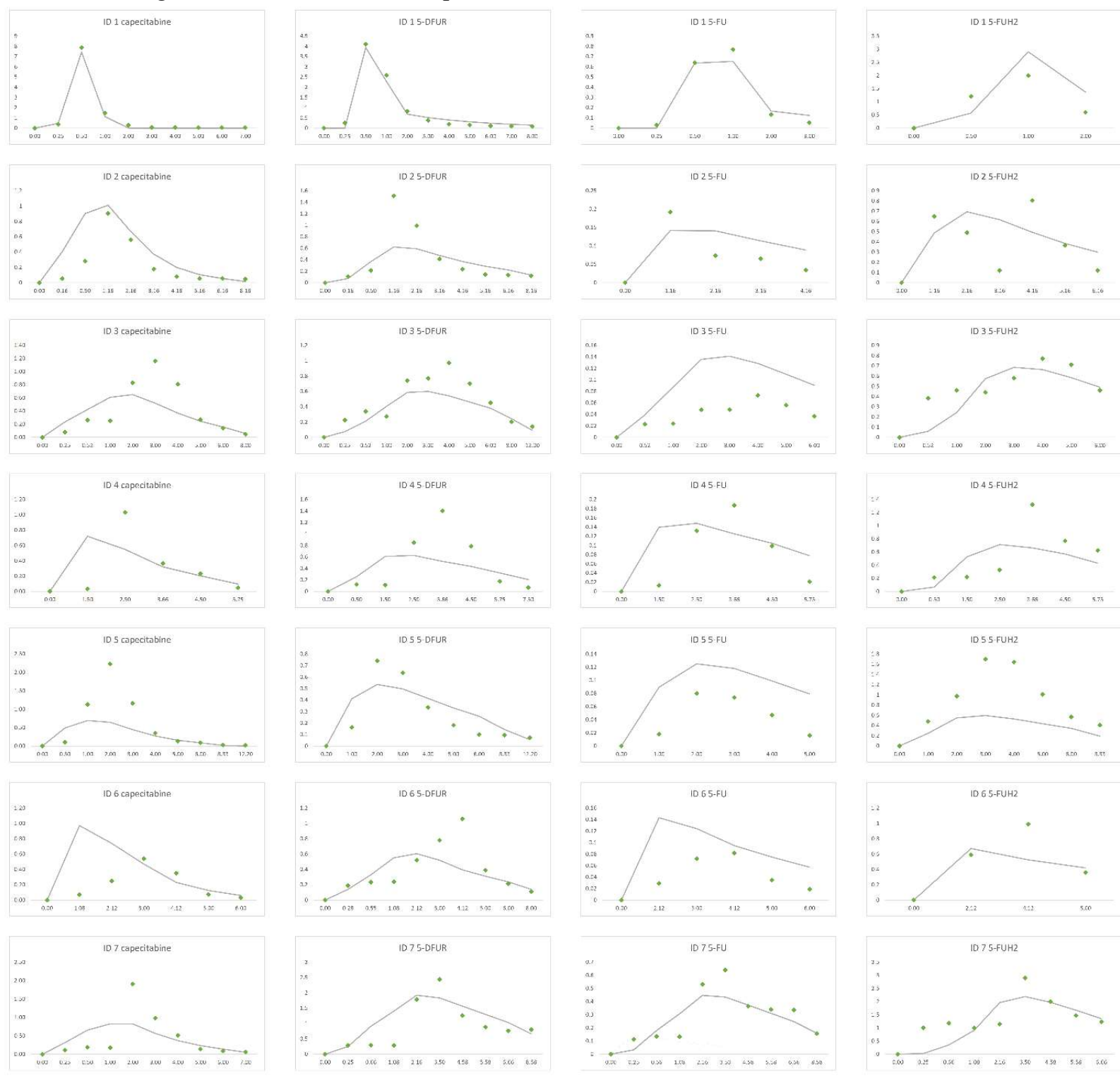

Figure 4. Individual predictions (gray) and observed data (green) of capecitabine, 5-DFUR, 5-FU and 5-FUH2 (columns 1-4) for each patient (rows 1-7). X label: time (hours). Y label: plasma concentrations ( $\mu \mathrm{g} / \mathrm{mL})$.

\section{DISCUSSION}

This work demonstrated that even small samples of data are valid to extract useful knowledge in clinical practice. Specifically, from the information of only 7 patients with colorectal cancer who were administered capecitabine, a joint population pharmacokinetic model of capecitabine, 5-DFUR, 5-FU and 5-FUH2 was developed. In combination with Bayesian methods and simulation techniques, this model allows to individualize posology adjustments $(26,27)$. These data were also used to estimate the optimal time point for monitoring the concentration of capecitabine, enabling the maximization of the potential value of new collected data (28). All this knowledge, generated from a small clinical routine sample, will allow clinicians to optimize and personalize treatments based on capecitabine and to improve the quality of new data collected in the future.

Regarding the structure of the final model, capecitabine, 5-FU and 5-FUH2 were described 

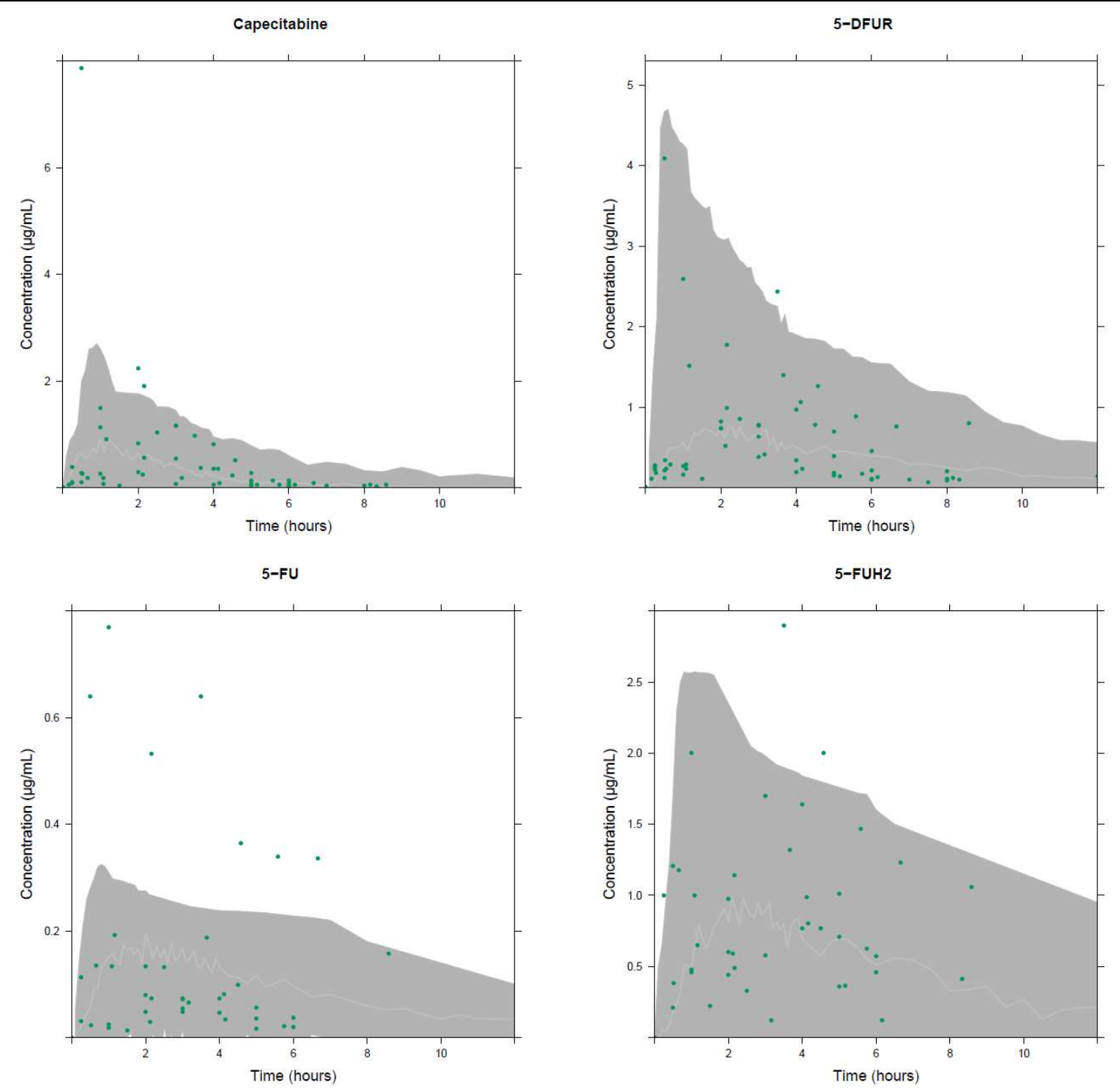

Figure 5. VPC for capecitabine, 5.DFUR, 5-FU and 5-FUH2. Green circles represent observed data. Dark grey areas represent the region between the lower and upper percentiles (5 and 95\%). Light grey lines represent the median.

with a single compartment and 5-DFUR with two compartments, assembling a multicompartmental model that described jointly the four compounds. This drug has been characterized using compartmental pharmacokinetic models by several authors $(10-12,29)$. In these works, capecitabine was described using a monocompartmental model with a first order absorption constant and a lag time. The metabolites were described by means of a linear series of monocompartmental models.

Among the cited works, our model resembled mostly the one by Urien et al. (12) and the ones by Blesch et al. (29) and Gieschke et al. (10). Specifically, our model described capecitabine in accordance with the model by Urien et al. The obtained population parameters for the model by
Urien et al. and our model had on average 2.07 and $0.995 \mathrm{~h}^{-1}$ for $k_{\mathrm{a}}, 0.28$ and $0.0172 \mathrm{~h}$ for $t_{\mathrm{lag}}, 338$ and $423 \mathrm{~L}$ for $V_{1}, 218$ and $396 \mathrm{~L} / \mathrm{h}$ for $C l_{0}$ and 12.9 and $4.05 \mathrm{~L} / \mathrm{h}$ for $C l_{\text {cap }}$, respectively. In our model, RSE values for $t_{\text {lag }}, \mathrm{Cl}_{23}$ and $\mathrm{Cl}_{32}$ were quite high $(87.9 \%$, $73.39 \%$ and $53.83 \%$, respectively), which could be due to sample size. Regarding the metabolites, although many works characterize 5-DFUR with a single compartment, our model described this metabolite with two compartments. Moreover we fixed the volumes of the metabolites to 1 in order to achieve a successful convergent model and, hence, the comparison of the obtained population parameters with those of other models was problematic. However, the ratios of volume to clearance $(\mathrm{V} / \mathrm{Cl})$ of Blesch et al. and Gieschke et 


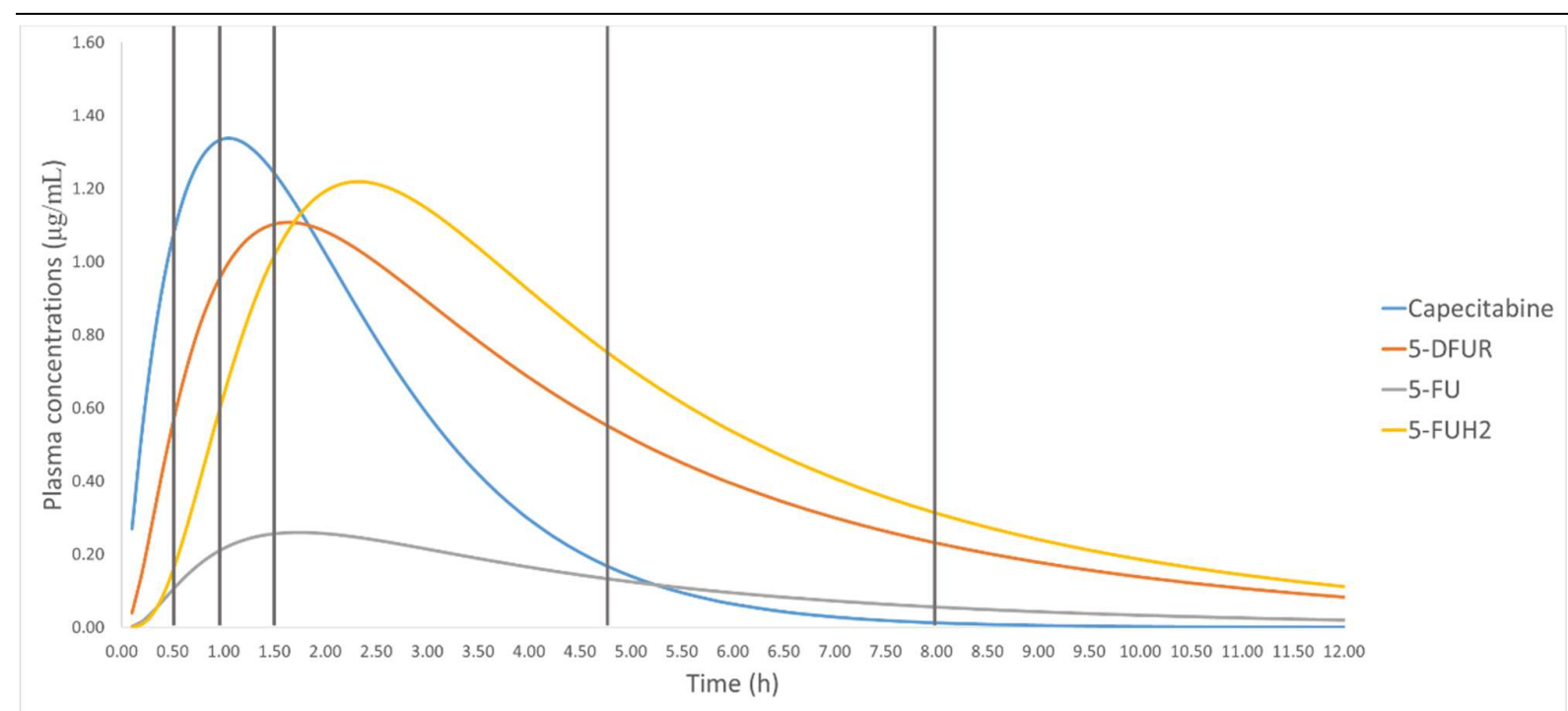

Figure 6. Population profiles for capecitabine, 5-DFUR, 5-FU and 5-FUH2 simulations and optimal sampling times $(0.546,0.892,1.562,4.736$ and 8 hours) after capecitabine administration.

al. were similar to ours. The obtained ratios for Blesch et al., Gieschke et al. and our model were $1.19,1.15$ and $0.39 \mathrm{~h}$ for $V_{2} / C l_{5 \text {-DFUR }}$ and 0.01 , 0.014 and $0.09 \mathrm{~h}$ for $V_{4} / C_{5-\mathrm{Fu}}$, respectively. A particularity of our model with respect to those of the literature was that the last characterized metabolite was 5-FUH2, as opposed to the rest of the discussed models which characterized FBAL.

One of the limitations of the study was the small sample size. Due to this fact, the model was not able to estimate all the necessary parameters to characterize four molecules simultaneously and, therefore, it was necessary to fix the volumes of the metabolites to achieve a successful convergent model. This technique has been used by other authors and is supported in small samples (30-32).

On the other hand, optimal sampling times were estimated using an auxiliary model that correctly adjusted the active metabolite 5-FU, responsible for the effect and toxicities derived from capecitabine. This model contained $91.94 \%$ of the observations within the $90 \%$ confidence interval of 1000 Monte Carlo simulations. The estimated optimal sampling times were 0.546 , $0.892,1.562,4.736$ and 8 hours after capecitabine administration. Rounding the times to achieve realistic sampling times for health personnel, the optimal sampling times can be established to be: $0.5,1,1.5,5$ and 8 hours after capecitabine administration. Capecitabine TDM is recommended specially for geriatric patients and those with hepatic dysfunction $(4,7,8)$.

To our knowledge, it is the first time that the optimal sampling times for capecitabine are estimated. Other authors have used a similar methodology to estimate optimal sampling times for other drugs. For example, Baille et al., (33), studied the optimal times of docetaxel and Jia et al., (28), for cyclosporin A in patients with heart transplantation.

\section{CONCLUSIONS}

We have established a joint multicompartmental pharmacokinetic model for capecitabine and its metabolites 5-DFUR, 5-FU and 5-FUH2 in patients with metastatic colorectal cancer. The carried out internal validation concludes that the proposed model describes the data adequately and, consequently, the application of Bayesian and simulation techniques will allow to predict concentration-time profiles of new patients from a single plasma sample.

Additionally, we have established the optimal sampling times of capecitabine, which are 0.546 , $0.892,1.562,4.736$ and 8 hours $(0.5,11.5,5$ and 8 hours, rounded) after capecitabine administration. Following these sampling times will maximize the PK information of new patients.

This work allows to conclude that even small samples of clinical routine data can contribute to the generation of useful knowledge to improve clinical care.

As a goal for future research, we intend to perform a TDM study at the proposed sampling times to make further improvements and externally validate the proposed population PK model. 


\section{ACKNOWLEDGMENTS}

This work is partially supported by "Ayuda para Doctorados Industriales del Ministerio de Economía, Industria y Competitividad" (Ref. DI15-07511).

\section{REFERENCES}

1. Hoff PM, Ansari R, Batist G, Cox J, Kocha W, Kuperminc M, et al. Comparison of Oral Capecitabine Versus Intravenous Fluorouracil Plus Leucovorin as First-Line Treatment in 605 Patients With Metastatic Colorectal Cancer: Results of a Randomized Phase III Study. J Clin Oncol. 2001;19(8):2282-92.

DOI: 10.1200/JCO.2001.19.8.2282

2. Van Cutsem E, Twelves C, Cassidy J, Allman D, Bajetta E, Boyer $\mathrm{M}$, et al. Oral Capecitabine compared with intravenous Fluorouracil Plus Leucovorin in Patients With Metastatic Colorectal Cancer : Results of a Large Phase III Study. J Clin Oncol. 2001;19(21):4097-106. DOI: 10.1200/JCO.2001.19.21.4097

3. Rea DW, Nortier JWR, Ten Bokkel Huinink WW, Falk S, Richel DJ, Maughan T, et al. A phase I/II and pharmacokinetic study of irinotecan in combination with capecitabine as first-line therapy for advanced colorectal cancer. Ann Oncol. 2005;16(7):1123-32.

10.1093/annonc/mdi227

4. Walko CM, Lindley C. Capecitabine: A Review. Clin Ther. 2005;27(1):23-44. DOI: $10.2307 / 2678832$

5. Miwa M, Ura M, Nishida M, Sawada N, Ishikawa $\mathrm{T}$, Mori $\mathrm{K}$, et al. Design of a novel oral fluoropyrimidine carbamate, capecitabine, which generates 5 fluorouracil selectively in tumours by enzymes concentrated in human liver and cancer tissue. Eur J Cancer. 1998;34(8):1274-81. DOI: 10.1016/S0959-8049(98)00058-6

6. Gieschke R, Burger H, Reigner B, Blesch KS, Steimer J. Population pharmacokinetics and concentration-effect relationships of capecitabine metabolites in colorectal cancer patients. Clin Pharmacol. 2003;55(November 2001):252-63. DOI: $\quad$ https://doi.org/10.1046/j.13652125.2003.01765.x

7. Paci A, Veal G, Bardin C, Levêque D, Widmer N, Beijnen J, et al. Review of therapeutic drug monitoring of anticancer drugs part 1 - Cytotoxics. Eur J Cancer. 2014;50(12):2010-9. DOI: 10.1016/j.ejca.2014.04.014

8. Walko CM, McLeod HL. Personalizing medicine in geriatric oncology. $\mathrm{J}$ Clin Oncol. 2014;32(24):2581-6.

DOI: 10.1200/JCO.2014.55.9047

9. Gerbrecht BM. Current Canadian experience with capecitabine: Partnering with patients to optimize therapy. Cancer Nurs. 2003;26(2):161-7. DOI: 10.1097/00002820-200304000-00011
10. Gieschke R, Reigner B, Blesch KS, Steimer JL. Population pharmacokinetic analysis of the major metabolites of capecitabine. J Pharmacokinet Pharmacodyn. 2002;29(1):25-47. DOI: 10.1023/A:1015716617967

11. Zandvliet A, Siegel-Lakhai W, Beijnen J, Copalu W, Etinne-Grimaldi M, Milano G, et al. PK/PD model of indisulam and capecitabine: interaction causes excessive myelosuppression. Clin Pharmacol Ther. 2008;83(6):829-39. DOI: 10.1038/sj.clp

12. Urien S, Rezaï K, Lokiec F. Pharmacokinetic modelling of 5-FU production from capecitabine A population study in 40 adult patients with metastatic cancer. J Pharmacokinet Pharmacodyn. 2005;32(5-6):817-33. DOI: 10.1007/s10928-0050018-2

13. Wakefield J, Racine-Poon A. An Application of Bayesian Population Pharmacokinetic/Pharmacodynamic Models To Dose Recommendation. Stat Med. 1995;14(9):971-86. DOI: $10.1002 /$ sim. 4780140917

14. Asuphon O, Montakantikul P, Houngsaitong J, Kiratisin P, Sonthisombat P. Optimizing intravenous fosfomycin dosing in combination with carbapenems for treatment of Pseudomonas aeruginosa infections in critically ill patients based on pharmacokinetic/pharmacodynamic (PK/PD) simulation. Int J Infect Dis. 2016;50:23-9. DOI: 10.1016/j.ijid.2016.06.017

15. Oteo I, Lukas JC, Leal N, Suarez E, Valdivieso A, Gastaca M, et al. Tacrolimus pharmacokinetics in the early post-liver transplantation period and clinical applicability via Bayesian prediction. Eur J Clin Pharmacol. 2013;69(1):65-74. DOI: $10.1007 / \mathrm{s} 00228-012-1300-\mathrm{z}$

16. Usman M, Frey OR, Hempel G. Population pharmacokinetics of meropenem in elderly patients: dosing simulations based on renal function. Eur J Clin Pharmacol. 2017;73(3):33342. DOI: $10.1007 / \mathrm{s} 00228-016-2172-4$

17. D’Argenio DZ. Optimal Sampling Times for Pharmacokinetic Experiments. J Pharmacokinet Biopharm. 1981;9(6). DOI: https://doi.org/10.1007/BF01070904

18. Hirzel A, Guisan A. Which is the optimal sampling strategy for habitat suitability modelling. Ecol Modell. 2002;157:331-41. DOI: https://doi.org/10.1016/S0304-3800(02)00203-X

19. Zufía L, Aldaz A, Giráldez J. Simple determination of capecitabine and its metabolites by liquid chromatography with ultraviolet detection in a single injection. J Chromatogr B Anal Technol Biomed Life Sci. 2004;809(1):51-8. DOI: 10.1016/j.jchromb.2004.06.004

20. Beal S, Sheiner L, Boeckmann A, Bauer R. NONMEM User's Guide. 1998.

21. Ribbing J, Jonsson EN. Power, Selection Bias and Predictive Performance of the Population Pharmacokinetic Covariate Model. J 
Pharmacokinet Pharmacodyn. 2004;31(2). DOI: 10.1023/B:JOPA.0000034404.86036.72

22. Steyerberg EW, Eijkemans MJC, Harrell Jr FE, Habbema JDF. Prognostic modelling with logistic regression analysis : a comparison of selection and estimation methods in small data sets. Stat Med. 2000;19(July 1999):1059-79. DOI: $10.1002 /($ SICI) $1097-$ 0258(20000430)19:8<1059::AIDSIM412>3.0.CO;2-0

23. Laupacis A, Sekar N, Stiell L. Clinical Prediction Rules: A Review and Suggested Modifications of Methodological Standards. JAMA. 1997;277(6):488-94. DOI: 10.1001/jama.1997.03540300056034

24. Gilks W, Richardson S, Spiegelhalter D. Markov Chain Monte Carlo in Practice. New York: Chapman and Hall/CRC.; 1996. 1-512 p. DOI: $10.1201 / \mathrm{b} 14835$

25. Dumont C, Lestini G, Le Nagard H, Mentré F, Comets E, Nguyen TT. PFIM 4.0, an extended R program for design evaluation and optimization in nonlinear mixed-effect models. Comput Methods Programs Biomed. 2018;156:217-29. DOI: 10.1016/j.cmpb.2018.01.008

26. Woillard JB, Debord J, Monchaud C, SaintMarcoux F, Marquet P. Population Pharmacokinetics and Bayesian Estimators for Refined Dose Adjustment of a New Tacrolimus Formulation in Kidney and Liver Transplant Patients. Clin Pharmacokinet. 2017;56(12):1491-8. DOI: $10.1007 / \mathrm{s} 40262-017-0533-5$

27. Flint RB, ter Heine R, Spaans E, Burger DM, de Klerk JCA, Allegaert K, et al. Simulation-based suggestions to improve ibuprofen dosing for patent ductus arteriosus in preterm newborns. Eur J Clin
Pharmacol. 2018;74(12):1585-91. DOI: 10.1007/s00228-018-2529-y

28. Jia Y, Meng XU, Li YAN, Xu C, Zeng WEN, Jiao $\mathrm{Y}$, et al. Optimal sampling time-point for cyclosporin A concentration monitoring in heart transplant recipients. Exp Ther Med. 2018;16:4265-70. DOI: 10.3892/etm.2018.6711

29. Blesch KS, Gieschke R, Tsukamoto Y, Reigner BG, Burger HU, Steimer J-L. Clinical pharmacokinetic/pharmacodynamic and physiologically based pharmacokinetic modeling in new drug development: the capecitabine experience. Invest New Drugs. 2003;21(2):195223. DOI: $10.1023 / \mathrm{A}: 1023525513696$

30. Wade JR, Kelman AW, Howie CA, Whiting B. Effect of misspecification of the absorption process on subsequent parameter estimation in population analysis. J Pharmacokinet Biopharm. 1993;21(2):209-22. DOI: 10.1007/BF01059771

31. Bauer RJ, Dedrick RL, White ML, Murray MJ, Garovoy MR. Population pharmacokinetics and pharmacodynamics of the anti-CD11a antibody hu1124 in human subjects with psoriasis. J Pharmacokinet Biopharm. 1999;27(4):397-420. DOI: 10.1023/A:1020917122093

32. Wählby U, Jonsson EN, Karlsson MO. Comparison of stepwise covariate model building strategies in population pharmacokinetic-pharmacodynamic analysis. AAPS PharmSci. 2002;4(4):68-79. DOI: 10.1208/ps040427

33. Schellens HM, Webster K, Rorer P, Raymond A, France PB, Maccallum P. Optimal Sampling Clearance Strategies for Bayesian Estimation of Docetaxel. Clin Cancer Res. 1997;3(September):1535-8. 